\title{
Mathematische Quilts
}

\author{
Katharina Habermann
}

Im letzten Heft der Mitteilungen (Heft 4-2008) waren die „Mathematischen Quilts“ als eines von zehn gelungenen Projekten im Jahr der Mathematik kurz vorgestellt worden. Im Rahmen einer Ausstellung waren sie erstmalig auf der DMVJahrestagung im September 2008 in Erlangen zu sehen und haben gezeigt, wie viel Begeisterung und neugieriges Interesse Mathematik auch bei Nichtmathematikern auszulösen vermag.

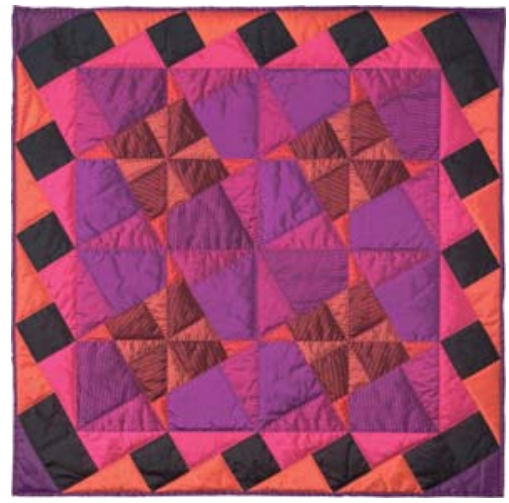

Hanni Strassberger: Moulin Rouge

Quilts sind mehr als eindrucksvolle Steppdecken. Ging es früher vielleicht einmal darum, aus Stoffen, Soffresten und einer flauschigen Füllung wärmende Decken oder Kleidung herzustellen, so steht heute vorrangig der dekorative Aspekt im Vordergrund.

Wer schon einmal in Amerika war, dem dürften diese textilen Wunderwerke nicht entgangen sein. Quilts werden dort als Kunst in Galerien und als historische $\mathrm{Ob}$ -

jekte in Museen ausgestellt. Und auch wenn man einen Fernseher besitzt, sind einem gewiss schon Quilts begegnet. Beim Zappen durch die Kanäle hat man gute Chancen bei einem amerikanischen Film eine Szene mit Quilt zu erwischen. Dazu muss man nicht in dem Film „Ein Amerikanischer Quilt" gelandet sein, in dem Freundinnen der Großmutter ihre Lebensgeschichte in einen Hochzeitsquilt für die Protagonistin einarbeiten. Man gewinnt schnell den Eindruck, als würden sich in Amerika ganze Familiengeschichten allein um Quilts ranken und in ihnen erzählt werden. In „Täglich grüßt das Murmeltier“ fallen mir auf Anhieb zwei Quilts ein, die Bettdecke und der Murmeltierquilt, der auf der großen Party an der Wand hängt. Auch in ,Independence Day“ oder ,Star Trek: Der Aufstand" wird man fündig. Und wenn Sie die Muße haben, einem $x$-beliebigen amerikanischen Film längere Zeit zu folgen: Spätenstens wenn ein Kind zu Bett gebracht wird, wird es mit einer gequilteten Bettdecke fürsorglich zugedeckt, oder wenn es sich jemand vor einem Kamin gemütlich macht, mummelt sich die Person in einen Quilt ein. Teilnehmer der DMV-Jahrestagung im vergangen Jahr in Erlangen hatten mit der Ausstellung „Mathematische Quilts“, die dort gezeigt worden ist, eine gute Gelegenheit kennenzulernen, was ein Quilt ist und welche Faszination von diesen textilen Kunstwerken ausgeht.
Bevor ich zu den „Mathematischen Quilts“ komme, vorab kurz etwas zu Quilts allgemein und Mathematik in Quilts. Ein Quilt besteht aus zwei Lagen Stoff (Oberund Rückseite) und einer dazwischenliegenden Wattierung, die durch gesteppte Nahtlinien miteinander verbunden werden. Die Ober- bzw. Schauseite wird dabei meist aus vielen Stoffeinzelteilen zu einem Patchwork (,Flickenwerk") zusammengesetzt. Für diese Patchworkoberteile eines Quilts haben sich in der Geschichte zahlreiche traditionelle Muster entwickelt, in denen aus mathematischer Perspektive sehr viel Geometrie und Symmetrie enthalten ist. So wie man beim Spazierengehen bei genauer Betrachtung des Bodens unter den Füßen bei kunstvoll angeordneten Pflastersteinen begeistert die Mathematik in den Mustern und Arrangements feststellen kann, so gibt es Patchwork-Quilt-Bücher, die sich wie mathematische Abhandlungen über Symmetriegruppen lesen, z. B. [2]. Und umgekehrt kann einer Quilterin beim Lesen eines Mathematikbuches über Parkettierungen, z. B. [3], ein um der andere Quilt vor dem geistigen Auge erscheinen. Auch andere mathematische Fachtexte sind pure Inspirationsquellen für Quiltmuster. Zumindest stecken in fast jedem Quilt Mathematik und mathematisches Arbeiten der Quilterin. Insbesondere beim Entwurf und der Umsetzung klassischer Quiltmuster sind grundlegende geometrische Kenntnisse unentbehrlich. Da das Nähen von Patchwork-Quilts auch heute noch fast ausschließlich eine reine Frauenangelegenheit ist, sind Patchwork-Quilts überdies ein gutes Beispiel für von Frauen betriebene „praktische“ Mathematik.

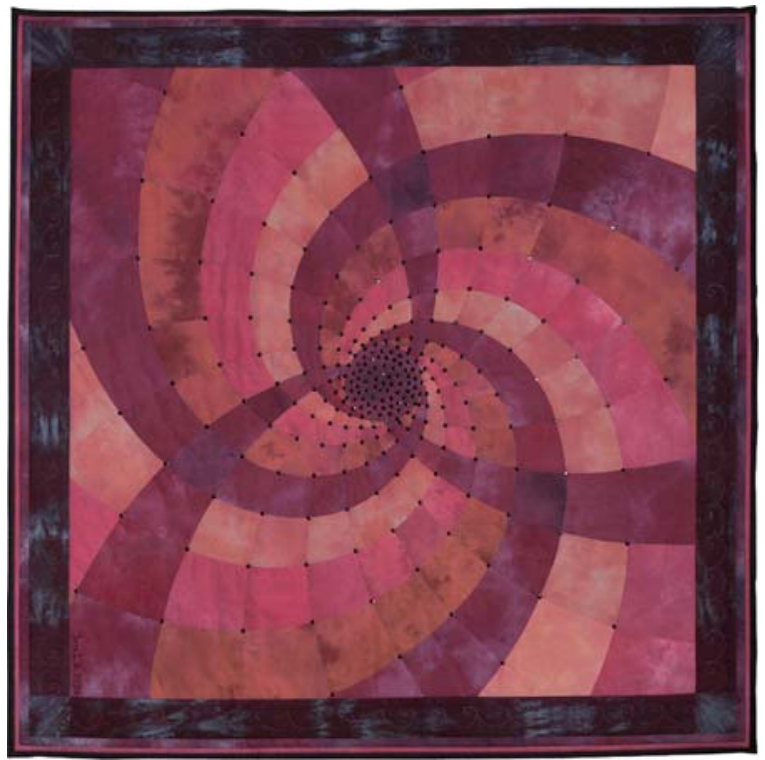

Irmi Karkat: 100I Versuch 


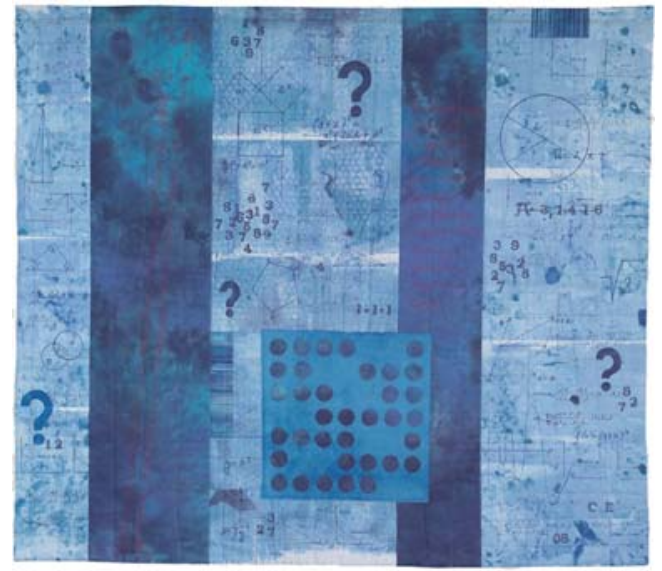

Christa Ebert: Ars Mathematica

Für das Jahr der Mathematik hatte ich die Idee, noch einen Schritt weiterzugehen und die Mathematik selbst zum Gegenstand der Gestaltung von Quilts zu machen. Ideen dazu ergeben sich von ganz allein. Da ich jedoch selbst nie die Zeit hätte aufbringen können, auch nur einen Bruchteil davon zu realisieren, kam ich auf den Gedanken, dass dies ein schönes und passendes Thema für eine Ausschreibung sei und dass sich gewiss Quilterinnen fänden, die sich für die Mathematik als Thema begeistern könnten.

In der Patchworkgilde Deutschland e.V. habe ich eine offene Ansprechpartnerin gefunden, die sich für meinen Vorschlag erwärmen konnte und ihn in Abstimmung mit der DMV als Projekt für das Jahr der Mathematik aufgriff. Mit einer Ausschreibung „Mathematische Quilts“ wurde schließlich im Dezember 2007 zum Nähen von Quilts aufgerufen, die mathematische Sachverhalte und Motive selbst thematisieren, die von mathematischen Ideen inspiriert worden sind oder zum Beispiel Aspekte aus der Geschichte der Mathematik aufgreifen. Innerhalb eines halben Jahres wurde dann offenbar mit großen Enthusiasmus - ohne Freude an Mathematik hätte man das in diesem kurzen Zeitraum gar nicht bewerkstelligen können - an den Quilts gearbeitet. Bis zum Sommer 2008 waren 93 Quilts eingereicht worden. Als Jurymitglied war ich von der Fülle der Ideen und noch mehr vom mathematischen Gehalt der Quilts respektive dem mathematischen Sachverstand der Quilterinnen beeindruckt. Nach strengen Maßstäben, sowohl was die Mathematik angeht als auch die handwerklich-gestalterische Umsetzung der Arbeit, wurden 24 Quilts für die Ausstellung ausgewählt, die dann erstmalig auf der DMV-Jahrestagung 2008 in Erlangen zu sehen war.

Die Vielfalt der gezeigten Quilts ist beachtlich. Fraktale waren ein naheliegendes Thema. So findet sich in der Ausstellung ein sehr schöner Quilt mit der Kochschen Schneeflockenkurve und ein Mengerteppich. Die Fibonacci-Zahlenfolge wurde gleich in mehren Quilts und auf unterschiedlichste Weise thematisiert. Ein anderer Quilt codiert das „Hohelied der Liebe“, ein weiterer die Verteilung der Primzahlen. Lottozahlen und Gewinn- quoten beim Lottospielen, das Pascalsche Dreieck und Mathematiker mit ihren bekanntesten Resultaten waren weitere Inhalte. Ein Quilt zeigt sogar einen Beweis: und zwar für den Satz des Pythagoras. Und eine Quilterin schien von den Schmierzetteln eines Mathematikers zu ihrem Quilt angeregt worden zu sein.

Wer Lust bekommen hat, sich seinen eigenen mathematischen Quilt zu nähen oder nähen zu lassen und klein anfangen möchte: in dem Buch „Making mathematics with needlework“ [I] (das Kapitel „,(k)not cables, braids“ aus diesem Buch wurde im Heft 16.1/2008 der DMV-Mitteilungen abgedruckt) findet man eine ausführliche Anleitung zum Nähen gequilteter Möbiusbänder.

Für alle diejenigen, welche die „Mathematischen Quilts“ in Erlangen nicht gesehen haben: Die Ausstellung wird auch im laufenden Jahr gezeigt. Der nächste bereits feststehende Termine ist das Bodensee-Quiltfestival vom 21 . bis zum 24. Mai 2009 in Radolfzell (Kulturzentrum Milchwerk).

\section{Literatur}

[1] Sarah-Marie Belcastro, Carolyn Yackel: Making mathematics with needlework. A K Peters (2008)

[2] Jinny Beyer: Tessellation-Quilts entwerfen: das Geheimnis der ineinandergreifenden Muster. Schäfer Hannover (200I)

[3] Hans-Günther Bigalke, Heinrich Wippermann: Reguläre Parkettierungen: mit Anwendungen in Kristallographie, Industrie, Baugewerbe, Design und Kunst. BI Wissenschaftsverlag Mannheim u. a. (1994)

Katharina Habermann, Nds. Staats- und Universitätsbibliothek Göttingen, Platz der Göttinger Sieben, 37073 Göttingen. habermann@sub.uni-goettingen.de

Die Ausstellung ist auch wunderbar dazu geeignet, an verschiedenen Orten gezeigt zu werden; die Patchworkgilde freut sich über Einladungen.

Ansprechpartnerin für die Ausstellung ist die Patchworkgilde Deutschland e.V. über ihre Vorsitzende Claudia Eichert-Schäfer (claudia@patchworkgilde.de). Bei der Patchworkgilde wird auch an der Erstellung einer CD mit Bildern und Beschreibungen der Quilts gearbeitet.

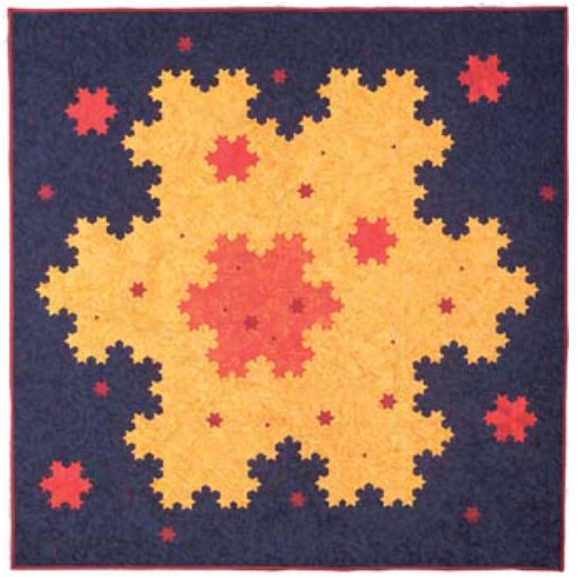

Constanze Hofmann: Kochs Sterne 